\title{
Therapeutic Uses of Triphala in Ayurvedic Medicine
}

\author{
Christine Tara Peterson, $\mathrm{PhD}^{1,2}$ Kate Denniston, $\mathrm{BS}^{3}$ and Deepak Chopra, $\mathrm{MD}^{1,2}$
}

\begin{abstract}
Aim: The aim of this article is to review the current literature on the therapeutic uses and efficacy of Triphala. Herbal remedies are among the most ancient medicines used in traditional systems of healthcare such as Ayurveda. Triphala, a well-recognized and highly efficacious polyherbal Ayurvedic medicine consisting of fruits of the plant species Emblica officinalis (Amalaki), Terminalia bellerica (Bibhitaki), and Terminalia chebula (Haritaki), is a cornerstone of gastrointestinal and rejuvenative treatment.

Methods: A search of the PubMed database was conducted.

Results: In addition, numerous additional therapeutic uses described both in the Ayurvedic medical literature and anecdotally are being validated scientifically. In addition to laxative action, Triphala research has found the formula to be potentially effective for several clinical uses such as appetite stimulation, reduction of hyperacidity, antioxidant, anti-inflammatory, immunomodulating, antibacterial, antimutagenic, adaptogenic, hypoglycemic, antineoplastic, chemoprotective, and radioprotective effects, and prevention of dental caries. Polyphenols in Triphala modulate the human gut microbiome and thereby promote the growth of beneficial Bifidobacteria and Lactobacillus while inhibiting the growth of undesirable gut microbes. The bioactivity of Triphala is elicited by gut microbiota to generate a variety of anti-inflammatory compounds.

Conclusions: This review summarizes recent data on pharmacological properties and clinical effects of Triphala while highlighting areas in need of additional investigation and clinical development.
\end{abstract}

Keywords: Ayurveda, anti-inflammatory, immunomodulating, microbiota, antioxidant, antimicrobial

\section{Introduction}

$\mathbf{H}$ ERBAL REMEDIES REPRESENT SOme of the most ancient medicines in healthcare and are historically considered among the most powerful means of maintaining human health and homeostasis. Ayurveda, a Sanskrit word meaning the knowledge of life or the science of perfect health, is the traditional system of personalized medicine from India, which emphasizes disease prevention and health promotion. Triphala (Sanskrit; tri $=$ three and phala=fruits) is a wellrecognized and revered polyherbal medicine consisting of dried fruits of the three plant species Emblica officinalis (Family Euphorbiaceae), Terminalia bellerica (Family Combretaceae), and Terminalia chebula (Family Combretaceae) that are native to the Indian subcontinent. It is classified as a tridoshic rasayana in Ayurvedic medicine as it promotes longevity and rejuvenation in patients of all constitutions and ages.

The formula consists of the fruits Amalaki or the Indian Gooseberry, Bibhitaki, and Haritaki of the three plants generally in equal proportions and has been used in traditional medicine in India for over 1000 years according to the writings of the great physician Charak in a foundational text of Ayurveda called the Charaka Samhita as well as in another key text called the Sushruta Samhita. According to Charak, taking the Triphala Rasayana (Triphala with honey and ghee) daily has the potential to make a person live for one hundred years devoid of old age and diseases. ${ }^{1}$ The physician Sushrut indicated that the formula is useful for treating ulcers and wounds. ${ }^{2}$

\footnotetext{
${ }^{1}$ Department of Family Medicine and Public Health, UC San Diego School of Medicine, Center of Excellence for Research and Training in Integrative Health, La Jolla, CA.

${ }^{2}$ Chopra Foundation, Department of Ayurveda and Yoga Research, Carlsbad, CA.

${ }^{3}$ Department of Naturopathic Medicine, Bastyr University, San Diego, CA.

(C) Christine Tara Peterson et al., 2017; Published by Mary Ann Liebert, Inc. This is an Open Access article distributed under the terms of the Creative Commons Attribution License, which permits unrestricted use, distribution, and reproduction in any medium, provided the original work is properly cited.
} 
As both Ayurveda and Western medicine agree that health and disease begin in the gut, ${ }^{1,3}$ Triphala represents an essential foundational formula as it promotes efficient digestion, absorption, elimination, and rejuvenation. Numerous references in well-respected Ayurvedic medical texts make clear that Triphala is revered as a multiuse therapeutic and perhaps even panacea historically.

\section{Ayurvedic Classification}

Triphala is classified as a tridoshic rasayana, meaning that the energetics are appropriate for Vata, Pitta, and Kapha or all types of patients. Charak describes rasayanas as having the qualities of supporting strength and immunity. ${ }^{1}$ Given these qualities and the tonic energetics, Triphala can be considered for use in the very young, the infirmed, and the elderly. Other classical Ayurvedic classifications attributed to the formula are shukrala, digestive, mild laxative at normal doses, bowel tonic at low dose, purgative at high doses, carminative, expectorant, antispasmodic, and bronchodilator. In addition, myriad other uses are described both in the Ayurvedic medical literature and anecdotally.

\section{Ayurvedic Pharmacology}

Dravya guna or Ayurvedic pharmacology describes the attributes of herbs. The rasa or taste of Triphala is sweet, sour, pungent, bitter, and astringent; the only taste not contained within the formula is salty. The virya, or potency and action, is neutral, and the vipaka, or postdigestive effect of the formula, is sweet. Triphala has a prabhav, meaning special action or trophism, for all doshas (energetics and mind-body types) and thus is balancing for all doshas and constitutions. The gunas, or qualities, of Amalaki are heavy and dry, and both Haritaki and Bhibitaki are considered light and dry. ${ }^{4}$

\section{Therapeutic Uses and Studies}

Ayurvedic medicine uses Triphala as a pillar of gastrointestinal treatment; however, the complexity of the three $\mathrm{ra}$ sayanas, or rejuvenative herbs, in the formulation allows for many applications. Moreover, studies have validated a number of potential uses of Triphala, which include free radical scavenging, antioxidant, anti-inflammatory, immunomodulating, appetite stimulation, gastric hyperacidity reduction, dental caries prevention, antipyretic, analgesic, antibacterial, antimutagenic, wound healing, anticariogenic, antistress, adaptogenic, hypoglycemic, anticancer, hepatoprotective, chemoprotective, radioprotective, and chemopreventive effects. ${ }^{4}$ Triphala may also promote proper digestion and absorption of food, reduce serum cholesterol levels, improve circulation, relax bile ducts, prevent immunosenescence, maintain homeostasis of the endocrine system, and increase production of red blood cells and hemoglobin. ${ }^{4}$

The major constituents of the formula are the tannins, gallic acid, ellagic acid, and chebulinic acid, which are potent antioxidants that may account, at least in part, for the observed immunomodulatory activity of the formula. ${ }^{5-7}$ Triphala also contains other bioactive compounds such as flavonoids (e.g., quercetin and luteolin), saponins, anthraquinones, amino acids, fatty acids, and various carbohydrates. ${ }^{6}$ In addition, Triphala-derived polyphenols such as chebulinic acid are also transformed by the human gut microbiota into bioactive metabolites, which have demonstrated potential in vitro to prevent oxidative damage. ${ }^{8}$

\section{Triphala in gastrointestinal health}

Triphala is perhaps most well known for its use in general gastrointestinal health. Animal studies have shown that both aqueous and alcohol-based extracts of Triphala prevent diarrhea. ${ }^{9}$ Triphala also induces enteroprotective effects, which are likely due, at least in part, to the high antioxidant content. In a rodent model, Triphala replenished depleted protein in the intestinal villi of the brush border as well as glutathione and phospholipid levels; the formula simultaneously decreased myeloperoxidase and xanthine oxidase levels in intestinal epithelium. ${ }^{10}$ In rats, Triphala exerted a gastroprotective effect on stress-induced ulcer. ${ }^{11}$ One human clinical trial that investigated the use of Triphala in patients with gastrointestinal disorders reported that treatment reduced constipation, mucous, abdominal pain, hyperacidity, and flatulence while improving the frequency, yield, and consistency of stool. ${ }^{12}$ Triphala also reduced colitis in a mouse model, and the treatment effect was attributed to antioxidant effects and high levels of flavonoids contained in Triphala. ${ }^{13}$

\section{Stress-reducing potential of Triphala}

Stress-induced disorders such as anxiety represent the leading causes of adult disability worldwide. ${ }^{14}$ Stress is a state of disharmony caused by perceived threat that is counteracted by an adaptive response to reestablish homeostasis and is associated with many chronic diseases. Animal studies have shown that Triphala protected against cold-induced stress and reversed stress-induced behavioral alterations and biochemical changes such as increased lipid peroxidation and corticosterone levels. ${ }^{15}$ Triphala also prevented noise-induced stress. ${ }^{16}$ In rats, Triphala prevented the noise-induced metabolic changes by mediating the antioxidant and cell-mediated immune response, and it was hypothesized that the biological mechanism is related to its antioxidant properties. ${ }^{6,16-19}$ Modern humans experience high levels of stress, thus adaptogenic treatments are needed more extensively in clinical practice.

\section{Antiobesogenic and antidiabetic potential of Triphala}

Deregulation of eating behavior is common in industrialized countries. Studies have demonstrated the potential of Triphala as a therapeutic agent for weight loss and reduction of body fat. In an animal study, Triphala was administered for 10 weeks to diet-induced obese mice. ${ }^{20}$ Triphala treatment decreased the percentage of body fat, body weight, and energy intake. Triphala also decreased total cholesterol, triglycerides, and low-density lipoprotein cholesterol in the experimental group compared with the control group. In a 12-week, double-blind, randomized placebo-controlled trial, human subjects treated with Triphala lost $\sim 5 \mathrm{~kg}$ compared with the placebo control group. ${ }^{21}$ Mean fasting blood sugar and fasting serum insulin levels were also reduced in the treated compared with control subjects. Given the global obesity epidemic, more treatment options are necessary to reduce the associated healthcare burden.

Triphala exerts hypoglycemic effects. Patients with type 2 diabetes are likely to have high postprandial blood glucose levels, especially after consuming carbohydrates. Elevated blood glucose results from the breakdown of carbohydrates by the digestive enzymes, alpha-amylase and alpha-glucosidase, 
and the reduced ability of cells to take in glucose from the blood. Past studies report that Triphala may exert actions similar to diabetic pharmaceutical drugs by inhibiting digestive enzymes and may decrease absorption of glucose through inhibition of glycolytic enzymes, thereby reducing blood glucose levels. One study demonstrated the inhibitory potential of Triphala on pancreatic glycolytic enzymes, namely alpha-amylase and alpha-glucosidase, which break down larger polysaccharides into glucose molecules that enter the blood stream. ${ }^{20}$

The role that Triphala plays in inhibiting starch digestion and absorption, thereby decreasing postprandial hyperglycemia, is similar to that of diabetes pharmaceutical drugs, such as miglitol and acarbose, which also target these glycolytic enzymes. In addition, Triphala decreased serum glucose levels in normal and alloxan-induced diabetic rats. ${ }^{22}$ A clinical study of noninsulin-dependent diabetes mellitus patients revealed that supplementation with $5 \mathrm{~g}$ of Triphala powder for 45 days significantly lowered blood glucose levels. ${ }^{23}$ Both fasting and postprandial blood glucose were reduced, which may be due to active ingredients such as sorbitol. Constituents in Triphala, including ellagitannins and gallotannins, also enhance both PPAR-alpha and gamma signaling, which increase insulin responsiveness and glucose uptake without inducing adipogenesis. ${ }^{24}$ These polyphenols may also promote decreased blood glucose and insulin levels in diabetic patients.

Triphala may also protect diabetics and those predisposed to diabetes through inhibition of glycation enzymes. Elevated blood glucose can cause severe damage through the process of glycation, in which sugar molecules compromise protein molecules in the body, which may in turn lead to nerve damage or blindness. Due to the presence of tannins, Triphala extract was found to effectively inhibit protein glycation in vitro. ${ }^{25}$ Triphala may also prevent glycation through promotion of lower blood glucose levels. As diabetes is the most prevalent endocrine disease globally, increased access to complementary hypoglycemic therapies for integrative care is needed.

\section{Triphala and cardiovascular health}

Cardiovascular disease is a leading cause of mortality and morbidity worldwide, and hypercholesteremia is an important risk factor. Animal studies have reported the hypercholesteremic effects of Triphala. In one study, Triphala reduced the total cholesterol, low-density lipoprotein, very low-density lipoprotein, and free fatty acid levels in rats fed an atherogenic diet for 48 days. ${ }^{26}$ Another study in rats fed an atherogenic diet revealed that Haritaki, one of the herbs in Triphala, induced hypolipidemic effects in the herbtreated group. A reduction in total cholesterol, triglycerides, and total protein and elevation of high-density lipoprotein cholesterol were found in the herb-treated group compared with control group. ${ }^{27}$ Triphala is a powerful herb to address imbalances in the gastrointestinal tract and cardiovascular system and should be more widely studied in the context of these common diseases.

\section{Antimicrobial potential of Triphala}

Overadministration of antibiotics has led to widespread drug resistance, thus it is becoming imperative that clinical researchers discover alternative and adjunctive antimicrobial agents with high efficacy. Both Triphala water decoctions (12\%) and ethanol extracts (14\%) have demonstrated antibacterial activity in vitro against bacterial isolates derived from patients infected with human immunodeficiency virus $^{28}$; the ethanol extracts were reported to have greater in vitro antimicrobial action against these species compared with the aqueous extracts, which may indicate lower solubility of the aromatic antibiotic compounds.

In addition, other studies report that these extracts also exert broad-spectrum antimicrobial action against antibioticresistant bacteria isolated from human subjects. The aqueous extracts (1:6) have demonstrated greater efficacy compared with the ethanol extracts (1:6) on pathogenic bacteria such as Escherichia coli and Staphylococcus aureus. ${ }^{29}$ In vitro, ethanol extracts of Triphala $(100 \mathrm{mg} / \mathrm{mL})$ components exhibited specific antimicrobial activity against multidrugresistant clinical bacterial isolates. ${ }^{30}$ Thus, Triphala was reported to exert antibacterial effects on both gram-positive and gram-negative species in vitro and demonstrated potential for further investigation as a complementary or adjunct antimicrobial therapy.

In addition to antimicrobial effects against oral bacteria, Triphala has also demonstrated the potential to eradicate enteric pathogens in vitro. One study tested the effects of Triphala aqueous extract $(200 \mathrm{mg} / \mathrm{mL})$ against enteric bacterial pathogens in vitro and found that Triphala possesses strong antibacterial effects against Staphylococcus epidermidis and S. aureus and moderate effects against Proteus vulgaris, Pseudomonas aeruginosa, and Salmonella typhi. ${ }^{30}$ In addition to antibacterial effects, Triphala is also known to exhibit antifungal properties. Triphala exerts antifungal action against Asperigillus species and has been reported to inhibit the fungus by up to $37.96 \%$ in vitro. In particular, the aqueous extracts $(1: 1)$ of fresh fruits were found to be more effective than dry fruits. ${ }^{31}$ Thus, the formula represents a promising antimicrobial candidate in need of further study.

The Potential of Triphala in Oral Care: Triphala has been used traditionally in Ayurvedic medicine as an antimicrobial agent. ${ }^{32}$ Numerous controlled clinical trials have shown that Triphala significantly reduces the abundance of oral bacteria, dental plaque, and gingivitis in human subjects. ${ }^{33-38}$ For example, Triphala is effective against Enterococcus faecalis, one of the most difficult to eliminate oral pathogens that are commonly isolated in chronic periodontitis. One study revealed that Triphala $(5 \mathrm{mg} / \mathrm{mL})$ in $10 \%$ dimethyl sulfoxide (DMSO) was equally or more effective at eliminating $E$. faecalis in vitro compared with $\mathrm{NaOCl}$, a common irrigant used during root canals. ${ }^{39}$ Triphala was more effective than $0.5 \%$ and $1 \% \mathrm{NaOCl}$ solution, but equally effective as $2.5 \%$ and $5 \%$ solutions.

In addition, another group reported that Triphala in $10 \%$ DMSO was more effective than $5.25 \% \mathrm{NaOCl}$ solution against $E$. faecalis biofilms on ex vivo tooth substrate and suggested the extract as an alternative in the context of clinical root canal irrigation. ${ }^{40,41}$ A study using human primary teeth ex vivo revealed that Triphala suspended in 10\% DMSO (1:3) exhibited significant antibacterial activity compared with control as well as higher antibacterial activity compared with $3 \% \mathrm{NaOCl}^{42}$ In vitro studies using Triphala ethanol extract have reported similar antimicrobial effects against $E$. faecalis compared with $2.5 \% \mathrm{NaOCl}^{43}$ Thus, Triphala may represent a potential therapy to eliminate 
E. faecalis as more side-effects and larger risk are associated with $\mathrm{NaOCl}$ solution treatment.

Dental caries, or cavities of the teeth, is a common infection associated with humans. Examining the use of Triphala as an oral antimicrobial agent, one study reported that Triphala dissolved in $10 \%$ DMSO exhibited a significant antibacterial effect ex vivo on extracted human mandibular premolars against Streptococcus mutans, which is one of the most prevalent oral pathogens responsible for dental cavities. ${ }^{44}$

Human clinical trials using Triphala water decoction as a mouthwash report that it exerts comparable efficacy compared with chlorhexidine gluconate germicidal mouthwash in the prevention of dental caries. ${ }^{45,46}$ For example, a study in human subjects revealed that Triphala $(6 \%)$ mouthwash promoted a significant reduction in oral streptococcus colonies. Oral streptococcus levels were measured after using a $6 \%$ Triphala mouthwash or $0.2 \%$ chlorhexidine mouthwash twice per day for $48 \mathrm{~h}$ and for 7 days; Streptococcus levels were reduced by $17 \%$ and $44 \%$, respectively, in the Triphala-treated group. ${ }^{33}$ The researchers concluded that Triphala was as effective as $0.2 \%$ chlorhexidine mouthwash given that the results of the Triphala treatment were similar to the chlorhexidine-treated group. Another double-blind human clinical trial also reported significant reductions in oral streptococcus levels at 5 and $60 \mathrm{~min}$ after rinsing with $15 \mathrm{~mL}$ aqueous Triphala extract (10\%) mouthwash. ${ }^{47}$

In addition, a double-blind, randomized human clinical trial reported that Triphala (10\%) mouthwash is effective against dental plaque and gingivitis in teenagers. ${ }^{48}$ The study reported Triphala as equally effective in antiplaque and antigingivitis activity compared with chlorhexidine. Moreover, a clinical study in children on the effects of Triphala $(0.6 \%)$ mouthwash on dental plaque, gingival inflammation, and microbial growth also compared its efficacy with a commercially available chlorhexidine mouthwash. The results indicated that both the germicidal chlorhexidine $(0.1 \%)$ and Triphala mouthwash were equally effective in reducing plaque and increasing gingival health after 9 months; however, Triphala was more effective than chlorhexidine in reducing microbial cell counts. ${ }^{49}$ A double-blind, randomized clinical trial in young adults also compared the efficacy of Triphala $(0.6 \%)$ and chlorhexidine $(0.12 \%)$ mouthwash for 21 days and reported a similar reduction in both plaque and gingival scores for both the Triphala- and chlorhexidine-treated groups. ${ }^{50}$ Triphala mouthwash treatment has also shown promise to reverse precancerous oral lesions associated with tobacco use in young adults. ${ }^{51}$

In periodontal diseases, matrix metalloproteinases (MMPs) degrade extracellular matrix proteins in a spectrum of processes that include tissue remodeling such as the connective tissue destruction observed in periodontitis. Ex vivo Triphala studies using extracted gingival tissue have demonstrated a greater reduction of MMP-9 activity in patient-derived white blood cells treated with Triphala compared with patientderived cells treated with the standard antibiotic drug. ${ }^{52}$ In treated tissue extracts, Triphala $(1.5 \mathrm{mg} / \mathrm{mL})$ reduced MMP-9 activity by $77 \%$, while doxycycline $(300 \mu \mathrm{g} / \mathrm{mL})$ reduced MMP-9 activity by $59 \%$. Thus, MMP inhibitors are important adjunctive therapies in periodontitis treatment and Triphala may represent a candidate to investigate in greater detail in this context. In addition, given the observed effectiveness of Triphala mouthwash compared with standard treatment, ad- ditional clinical trials should be performed to identify the potential for integration in dentistry.

\section{Radioprotective effects of Triphala}

Studies have concluded that Triphala may help prevent and reverse DNA damage and mutagenesis. ${ }^{53}$ The prevention of DNA damage is important given that it is often an initiating event in carcinogenesis. Research in animal models and in vitro has shown that Triphala is effective in prevention of mutagenesis induced by both chemical- and radiationinduced damage. ${ }^{54-56}$ An in vitro study found that Triphala eliminated reactive oxygen species in HeLa cells exposed to ionizing X-radiation or bleomycin, both of which generate DNA strand breaks through the generation of reactive oxygen species. ${ }^{57}$ In addition, gamma-radiation-induced plasmid DNA strand break was inhibited by Triphala in vitro. The rasayana formulation also inhibited radiation-induced lipid peroxidation in rat liver microsomes and demonstrated the ability to scavenge free radicals such as superoxide. Importantly, the high levels of phenolic compounds such as gallic acid were attributed to the free radical scavenging activity. ${ }^{56}$

In animal models, Triphala intervention reduced radiationinduced mortality by $60 \%$ in mice fed Triphala for only 7 days before whole-body gamma-irradiation. ${ }^{54}$ Triphala reversed the increased xanthine oxidoreductase and decreased the superoxide dismutase activity that was observed post-irradiation. Treatment with Triphala also prevented DNA damage in murine white blood cells and spleen cells post-irradiation. Triphala may play a protective role against oxidation, even when administered after exposure.

In addition, other animal studies have reported significantly reduced acute intestinal damage after ionizing radiation exposure in groups fed Triphala $(1 \mathrm{~kg} / \mathrm{g})$ for at least 5 days before radiation treatment. ${ }^{58}$ Moreover, studies have demonstrated that Triphala feeding before gamma-radiation exposure reduced radiation sickness and mortality in mice. ${ }^{59}$ Triphala was reported to scavenge hydroxyl, superoxide anion, and nitric oxide free radicals in a dose-dependent manner in vitro. ${ }^{59}$ Triphala aqueous extract feeding for 5 days before exposure was also reported as protective against gammaradiation in mice at a dose of $10 \mathrm{mg} / \mathrm{kg}$, which was $1 / 28$ of the calculated LD50 dose. ${ }^{60}$ Thus, the antioxidant and free radical scavenging activities of Triphala were concluded to serve a role in its protective effect against ionizing radiation. Human clinical trials to further elucidate the mechanisms of radioprotective action and clinical utility are required.

\section{Antineoplastic activity of Triphala}

Triphala has been investigated as a potential antineoplastic agent. ${ }^{61}$ Numerous studies have been performed in this context and have shown that Triphala exerts an antineoplastic effect on many cancer cell lines, including those of the breast, prostate, colon, and pancreas. ${ }^{62-64}$ Data in cell lines show that Triphala has a differential modulatory effect on normal and cancer cell lines. Triphala induces cytotoxicity in cancer cells, which showed increases in intracellular reactive oxygen species, but not normal cells. Excised tumor tissue from Triphala-fed mice compared with controls suggested that apoptosis induction may have mediated reduced tumor growth. ${ }^{65}$

Preclinical studies using in vitro and in vivo models report that Triphala inhibits cancer growth in both cell and in vivo 
models and the effects are mediated through the ERK and p53 pathways. ${ }^{63}$ In addition, methanol extract of Triphala suppressed proliferation and induced $\mathrm{p} 53$-independent apoptosis in human colon cancer stem cells. ${ }^{64}$ Triphala suppressed the expression of oncogenes, c-Myc and Cyclin D1, and thus Wnt pathway signaling to reduce proliferation and resistance to apoptosis. Triphala-induced apoptotic induction occurred through the intrinsic mitochondrial apoptotic signaling pathway. Moreover, one clinical trial reported that Triphala powder treatment in healthy humans increased cytotoxic $\mathrm{T}$ cells and NK cells in the experimental group compared with the control group. ${ }^{66}$ Thus, Triphala shows potential as an antineoplastic agent and thus should be systematically explored for potential as an adjunct therapy in the management of colon and other cancers.

\section{Antioxidant activity of Triphala and eye health}

Antioxidant effects of Triphala have the potential to help maintain eye health. Triphala is a rich source of vitamin $\mathrm{C}$ and flavonoids. One study used Triphala as a pretreatment in selenite-induced cataracts in mice. Triphala significantly restored glutathione levels in eye lenses. Triphala also increased the activities of antioxidant enzymes, such as superoxide dismutase, catalase, glutathione- $S$-transferase, and glutathione peroxidase, in the lenses of the experimental group when compared with the control group. While $100 \%$ of the mice in the control group developed cataracts, only $20 \%$ of the mice pretreated with Triphala developed cataracts. This effect may be linked to the antioxidant activity of Triphala. ${ }^{67}$

\section{Anti-inflammatory effects of Triphala}

Chronic inflammation is deleterious and affects most major chronic health conditions. Triphala has shown promise as an anti-inflammatory agent. In one study, Triphala performed better or equivalent when compared with standard drug treatment for a variety of biochemical measurements of inflammation. ${ }^{19}$ In addition, Triphala significantly reduced inflammatory markers as well as bone and cartilage degradation in arthritic rats. ${ }^{68}$ In this study, Triphala extract was significantly more effective than the nonsteroidal anti-inflammatory drug, indomethacin, in ameliorating arthritic and inflammatory effects. Triphala reduced expression of inflammatory mediators such as IL-17, COX-2, and RANKL through inhibition of NF- $\mathrm{KB}$ activation. Another study found that Triphala increased antioxidant levels and decreased lipid peroxidation in the tissues of arthritic rats. ${ }^{69}$

In lipopolysaccharide-stimulated macrophages, Triphala treatment suppressed production of inflammatory mediators (such as TNF $\alpha$, IL-1 $\beta$, IL-6, MCP-1, VEGF, NO, and PGE2), intracellular free radicals, inflammatory enzymes (such as iNOS and COX-2), and lysosomal enzyme release. ${ }^{70}$ Chebulagic acid, a constituent in Triphala, was found to inhibit COX and 5-LOX, which are both major enzymes involved in inflammation and carcinogenesis. ${ }^{71}$ Triphala also increased antioxidant activity in mice after induction of nephrotoxicity from bromobenzene. Triphala ameliorated nephrotoxic effects by upregulating antioxidant enzymes, superoxide dismutase, glutathione- $S$ transferase, and glutathione peroxidase. Lipid peroxidation and markers of kidney dysfunction were reduced in the
Triphala-treated group compared with controls. ${ }^{72}$ The antiinflammatory effects of Triphala should be investigated in greater detail.

\section{Antiaging effects of Triphala}

Triphala extract exerted highly protective antiaging effects on human skin cells in vitro. Triphala extract affects gene expression of human skin cells, stimulating collagen-1 and elastin-synthesizing genes and antioxidant genes responsible for the cellular antioxidant, SOD-2. Triphala extract was found to inhibit melanin production and hyperpigmentation due to the presence of protective phytochemicals. Furthermore, Triphala extract exhibited significant free radical scavenging activity on hydrogen peroxideinduced cell damage and senescence. ${ }^{73}$ These results demonstrate potential dermal antiaging effects of Triphala, such as increasing collagen and elastin, increasing cellular antioxidants, and decreasing hyperpigmentation.

\section{Variable efficacy of herbal therapies}

A number of factors, including variability in herbal source, processing, bioavailability, digestion, and absorption of herbal components, cause the true efficacy of herbs on human health to be highly variable. This variability is known to be at least partially due to inherent variation in the gut microbiota that act on the ingested components of herbal remedies and transform them into compounds with increased bioabsorption and bioactivity. These features have confounded the true efficacy of herbal remedies as it pertains to the maintenance of human health and/or the ability to reverse chronic disease states.

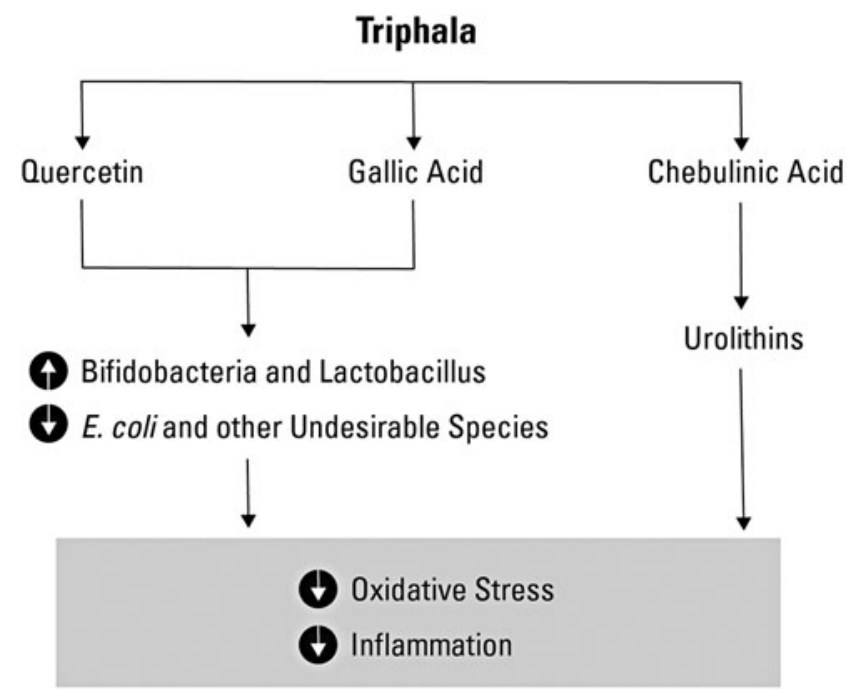

FIG. 1. The bioactivity of Triphala is elicited by gut microbiota. The phytochemicals in Triphala promote the growth of beneficial gut microbes such as Bifidobacteria and Lactobacillus species while inhibiting the growth of less desirable and potentially more inflammatory gut residents such as Escherichia coli. The enzymatic activity of lactic acid bacteria degrades tannins in Triphala such as gallic acid. Triphala-derived polyphenols such as chebulinic acid are also transformed by the human gut microbiota into metabolites such as urolithins, which have the potential to prevent oxidative damage and inflammation. Illustration by Victor Hewitt; used with permission. 
The increased popularity of herbal remedies such as Triphala has led to dramatic improvements in the processing of crude plant materials that serve to maximize the absorption of otherwise poorly absorbed plant components. Despite these improvements, these preparations still display pronounced variability in efficacy, which is likely related to the natural variation in composition of gut microbiota species that catalyze the biotransformation of herbal components. This response variability is not unique to herbs and, in fact, may be the case for virtually all health-promoting compounds ingested by humans (e.g., polyphenolic compounds derived from plants).

\section{Triphala and the gut microbiome}

It is known that phytochemicals in Triphala such as quercetin and gallic acid promote the growth of Bifidobacteria and Lactobacillus species while inhibiting the growth of undesirable gut residents such as E. coli. ${ }^{17,74-76}$ In addition, the lactic acid bacteria possess enzymatic activity (e.g., tannase) to degrade plant tannins such as gallic acid contained in Triphala. ${ }^{77-79}$ For example, Triphala-derived polyphenols such as chebulinic acid are transformed by the human gut microbiota into metabolites such as urolithins (Fig. 1), which have the potential to prevent oxidative damage. ${ }^{8}$ The authors speculate that the bioactivity of Triphala is elicited by the gut microbiome to generate a widened spectrum and abundance of anti-inflammatory compounds.

Triphala-induced benefits in both the elderly and persons of all ages may be enhanced by coadministration of specific probiotic species. Thus, probiotic formulations consisting of bacterial species capable of mediating the increased digestion, bioabsorption, and bioactivity of Triphala may increase and make more uniform the response and impact of Triphala treatment on human populations. Further studies are required to determine the full effect of Triphala on gut microbiota and the potential of specific probiotics to increase the efficacy of the herb.

\section{Conclusions}

Triphala is a powerful polyherbal formula with myriad efficacious therapeutic uses for maintaining homeostasis as well as the prevention and treatment of disease. Many scientific studies have reported evidence-based validation of various traditional uses of Triphala. It provides therapeutic value for multiple pathologies. Additional government funding allocations and support are needed for further and ongoing studies to validate its therapeutic uses in human clinical trials and to define the biological mechanisms relevant to this plant-based medicine. More widespread education of the general public and medical providers on clinical Ayurvedic medicine and complementary therapies such as Triphala is warranted to increase awareness of these treatments for both clinical and healthy populations.

\section{Author Disclosure Statement}

C.T.P. is a postdoctoral fellow at UCSD partially funded by the Chopra Foundation. D.C. is a founder of the Chopra Foundation and Chopra Center and a co-owner of the Chopra Center.

\section{References}

1. Agniveśa, Cakrapānidatta, Śarmā, RM, Dash B. Agniveaś's Caraka samhita: Text with English Translation \& Critical
Exposition Based on Cakrapāni Datta's $\bar{A}$ yurveda dīpik $\bar{a}$, 1st ed. Varanasi, India: Chowkhamba Sanskrit Series Office, 1976.

2. Bhishagratna K. An English Translation of the Sushruta Samhita, Based on Original Sanskrit Text, with a Full and Comprehensive Introd., Additional Texts, Different Readings, Notes, Comparative Views, Index, Glossary And Plates, 2nd ed. Varanasi, India: Chowkhamba Sanskrit Series Office, 1963.

3. Lloyd GER (ed). Hippocratic Writings, new ed. Chadwick J, Mann WN (translators). London, England: Penguin, 1978.

4. Baliga MS, et al. Scientific validation of the ethnomedicinal properties of the Ayurvedic drug Triphala: A review. Chin J Integr Med 2012;18:946-954.

5. Lu K, et al. Triphala and its active constituent chebulinic acid are natural inhibitors of vascular endothelial growth factor-a mediated angiogenesis. PLoS One 2012;7:e43934.

6. Belapurkar P, Goyal P, Tiwari-Barua P. Immunomodulatory effects of triphala and its individual constituents: A review. Indian J Pharm Sci 2014;76:467-475.

7. Lee HS, et al. Antioxidant effects of aqueous extract of Terminalia chebula in vivo and in vitro. Biol Pharm Bull 2005;28:1639-1644.

8. Olennikov DN, Kashchenko NI, Chirikova NK. In vitro bioaccessibility, human gut microbiota metabolites and hepatoprotective potential of chebulic ellagitannins: A case of Padma Hepaten ${ }^{\circledR}$ formulation. Nutrients 2015;7:8456-8477.

9. Biradar YS, et al. Evaluation of anti-diarrhoeal property and acute toxicity of Triphala Mashi, an Ayurvedic formulation. J Herb Pharmacother 2007;7:203-212.

10. Nariya M, Shukla V, Jain S, Ravishankar B. Comparison of enteroprotective efficacy of triphala formulations (Indian herbal drug) on methotrexate-induced small intestinal damage in rats. Phytother Res 2009;23:1092-1098.

11. Nariya MB, Shukla VJ, Ravishankar B, Jain SM. Comparison of gastroprotective effects of triphala formulations on stress-induced ulcer in rats. Indian J Pharm Sci 2011;73: 682-687.

12. Pulok K, Mukherjee SR, Bhattacharyya S, et al. Clinical study of 'Triphala' - A well known phytomedicine from India. Iran J Pharmacol Ther 2005;5:51-54.

13. Rayudu V, Raju AB. Effect of Triphala on dextran sulphate sodium-induced colitis in rats. Ayu 2014;35:333-338.

14. Whiteford HA, Ferrari AJ, Degenhardt L, et al. The global burden of mental, neurological and substance use disorders: An analysis from the Global Burden of Disease Study 2010. PLoS One 2015;10:e116820.

15. Dhanalakshmi S, Devi RS, Srikumar R, et al. Protective effect of Triphala on cold stress-induced behavioral and biochemical abnormalities in rats. Yakugaku Zasshi 2007; 127:1863-1867.

16. Srikumar R, Parthasarathy NJ, Manikandan S, et al. Effect of Triphala on oxidative stress and on cell-mediated immune response against noise stress in rats. Mol Cell Biochem 2006;283:67-74.

17. Carlsen $\mathrm{MH}$, et al. The total antioxidant content of more than 3100 foods, beverages, spices, herbs and supplements used worldwide. Nutr J 2010;9:3.

18. Kumari N, et al. Effects of ionizing radiation on microbial decontamination, phenolic contents, and antioxidant properties of triphala. J Food Sci 2009;74: M109-M113.

19. Rasool M, Sabina EP. Antiinflammatory effect of the Indian Ayurvedic herbal formulation Triphala on adjuvantinduced arthritis in mice. Phytother Res 2007;21:889-894. 
20. Gurjar S, Pal A, Kapur S. Triphala and its constituents ameliorate visceral adiposity from a high-fat diet in mice with diet-induced obesity. Altern Ther Health Med 2012; 18:38-45.

21. Kamali SH, et al. Efficacy of 'Itrifal Saghir', a combination of three medicinal plants in the treatment of obesity; A randomized controlled trial. Daru 2012;20:33.

22. Patel DK, Kumar R, Laloo D, Hemalatha S. Diabetes mellitus: An overview on its pharmacological aspects and reported medicinal plants having antidiabetic activity. Asian Pac J Trop Biomed 2012;2:411-420.

23. Rajan SS, Antony S. Hypoglycemic effect of triphala on selected non insulin dependent diabetes mellitus subjects. Ancient Sci Life 2008;27:45-49.

24. Yang MH, Vasquez Y, Ali Z, et al. Constituents from Terminalia species increase PPARalpha and PPARgamma levels and stimulate glucose uptake without enhancing adipocyte differentiation. J Ethnopharmacol 2013;149:490-498.

25. Ganeshpurkar A, Jain S, Agarwal S. Experimental studies on glycolytic enzyme inhibitory and antiglycation potential of Triphala. Ayu 2015;36:96-100.

26. Saravanan S, Srikumar R, Manikandan S, et al. Hypolipidemic effect of triphala in experimentally induced hypercholesteremic rats. Yakugaku Zasshi 2007;127:385-388.

27. Maruthappan V, Shree KS. Hypolipidemic activity of haritaki (Terminalia chebula) in atherogenic diet induced hyperlipidemic rats. J Adv Pharm Technol Res 2010;1: 229-235.

28. Srikumar R, et al. Evaluation of the growth inhibitory activities of Triphala against common bacterial isolates from HIV infected patients. Phytother Res 2007;21:476-480.

29. Biradar YS, Jagatap S, Khandelwal KR, Singhania SS. Exploring of antimicrobial activity of Triphala Mashi-An ayurvedic formulation. Evid Based Complement Altern Med 2008;5:107-113.

30. Tambekar DH, Dahikar SB. Antibacterial activity of some Indian ayurvedic preparations against enteric bacterial pathogens. J Adv Pharm Technol Res 2011;2:24-29.

31. Gautam AK, Avasthi S, Sharma A, Bhadauria R. Antifungal potential of triphala churna ingredients against $A s$ pergillus species associated with them during storage. Pak J Biol Sci 2012;15:244-249.

32. Prakash S, Shelke AU. Role of Triphala in dentistry. J Indian Soc Periodontol 2014;18:132-135.

33. Srinagesh J, Krishnappa P, Somanna SN. Antibacterial efficacy of triphala against oral streptococci: An in vivo study. Indian J Dent Res 2012;23:696.

34. Shanbhag VK. Triphala in prevention of dental caries and as an antimicrobial in oral cavity-A review. Infect Disord Drug Targets 2015;15:89-97.

35. Pradeep AR, et al. Triphala, a new herbal mouthwash for the treatment of gingivitis: A randomized controlled clinical trial. J Periodontol 2016;87:1352-1359.

36. Mamgain P, Kandwal A, Mamgain RK. Comparative evaluation of triphala and ela decoction with $0.2 \%$ chlorhexidine as mouthwash in the treatment of plaque-induced gingivitis and halitosis: A randomized controlled clinical trial. J Evid Based Complement Altern Med 2016; DOI:10.1177/ 2156587216679532.

37. Bhattacharjee R, et al. Efficacy of triphala mouth rinse (aqueous extracts) on dental plaque and gingivitis in children. J Investig Clin Dent 2015;6:206-210.

38. Naiktari RS, Gaonkar P, Gurav AN, Khiste SV. A randomized clinical trial to evaluate and compare the efficacy of triphala mouthwash with $0.2 \%$ chlorhexidine in hospitalized patients with periodontal diseases. J Periodontal Implant Sci 2014;44:134-140.

39. Shakouie $S$, et al. An in vitro comparison of the antibacterial efficacy of triphala with different concentrations of sodium hypochlorite. Iran Endod J 2014;9:287-289.

40. Prabhakar J, et al. Evaluation of antimicrobial efficacy of herbal alternatives (Triphala and green tea polyphenols), MTAD, and 5\% sodium hypochlorite against Enterococcus faecalis biofilm formed on tooth substrate: An in vitro study. J Endod 2010;36:83-86.

41. Agrawal V, Kapoor S, Agrawal I. Critical review on eliminating endodontic dental infections using herbal products. J Diet Suppl 2017;14:229-240.

42. Thomas S, Asokan S, John B, et al. Comparison of antimicrobial efficacy of diode laser, triphala, and sodium hypochlorite in primary root canals: A randomized controlled trial. Int J Clin Pediatr Dent 2017;10:14-17.

43. Saxena D, Saha SG, Saha MK, et al. An in vitro evaluation of antimicrobial activity of five herbal extracts and comparison of their activity with $2.5 \%$ sodium hypochlorite against Enterococcus faecalis. Indian J Dent Res 2015;26: 524-527.

44. Prabhakar J, Balagopal S, Priya MS, et al. Evaluation of antimicrobial efficacy of Triphala (an Indian Ayurvedic herbal formulation) and $0.2 \%$ chlorhexidine against Streptococcus mutans biofilm formed on tooth substrate: An in vitro study. Indian J Dent Res 2014;25:475-479.

45. Tandon S, Gupta K, Rao S, Malagi KJ. Effect of Triphala mouthwash on the caries status. Int J Ayurveda Res 2010;1: 93-99.

46. Srinagesh J, Pushpanjali K. Assessment of antibacterial efficacy of triphala against mutans streptococci: A randomised control trial. Oral Health Prev Dent 2011;9:387-393.

47. Saxena S, Lakshminarayan N, Gudli S, Kumar M. Anti bacterial efficacy of Terminalia chebula, Terminalia bellirica, Embilica officinalis and Triphala on salivary streptococcus mutans count-A linear randomized cross over trial. J Clin Diagn Res 2017;11:ZC47-ZC51.

48. Chainani $\mathrm{SH}$, et al. Antiplaque and antigingivitis efficacy of triphala and chlorhexidine mouthrinse among schoolchildrenA cross-over, double-blind, randomised controlled trial. Oral Health Prev Dent 2014;12:209-217.

49. Bajaj N, Tandon S. The effect of Triphala and Chlorhexidine mouthwash on dental plaque, gingival inflammation, and microbial growth. Int J Ayurveda Res 2011;2:29-36.

50. Baratakke SU, et al. Efficacy of triphala extract and chlorhexidine mouth rinse against plaque accumulation and gingival inflammation among female undergraduates: A randomized controlled trial. Indian J Dent Res 2017;28: 49-54.

51. Deshpande A, Tandon S, Deshpande N. Low resource screening method of pre-cancerous lesions and its reversal by Triphala in teen-age Indian population. Ayu 2014;35: 160-167.

52. Abraham S, Kumar MS, Sehgal PK, et al. Evaluation of the inhibitory effect of triphala on PMN-type matrix metalloproteinase (MMP-9). J Periodontol 2005;76:497-502.

53. Baliga MS, Meera S, Vaishnav LK, et al. Rasayana drugs from the Ayurvedic system of medicine as possible radioprotective agents in cancer treatment. Integr Cancer Ther 2013;12:455-463.

54. Sandhya $\mathrm{T}$, et al. Protection against radiation oxidative damage in mice by Triphala. Mutat Res 2006;609:17-25. 
55. Kaur S, Arora S, Kaur K, Kumar S. The in vitro antimutagenic activity of Triphala-An Indian herbal drug. Food Chem Toxicol 2002;40:527-534.

56. Naik GH, et al. In vitro antioxidant studies and free radical reactions of triphala, an ayurvedic formulation and its constituents. Phytother Res 2005;19:582-586.

57. Takauji Y, et al. Triphala, a formulation of traditional Ayurvedic medicine, shows protective effect against $X$-radiation in HeLa cells. J Biosci 2016;41:569-575.

58. Yoon WS, et al. Protective effect of triphala on radiation induced acute intestinal mucosal damage in Sprague Dawley rats. Indian J Exp Biol 2012;50:195-200.

59. Jagetia GC, Malagi KJ, Baliga MS, et al. Triphala, an ayurvedic rasayana drug, protects mice against radiationinduced lethality by free-radical scavenging. J Altern Complement Med 2004;10:971-978.

60. Jagetia GC, Baliga MS, Malagi KJ, Sethukumar Kamath M. The evaluation of the radioprotective effect of Triphala (an ayurvedic rejuvenating drug) in the mice exposed to gamma-radiation. Phytomedicine 2002;9:99-108.

61. Baliga MS. Triphala, Ayurvedic formulation for treating and preventing cancer: A review. J Altern Complement Med 2010;16:1301-1308.

62. Kaur S, Michael H, Arora S, et al. The in vitro cytotoxic and apoptotic activity of Triphala-An Indian herbal drug. J Ethnopharmacol 2005;97:15-20.

63. Shi Y, Sahu RP, Srivastava SK. Triphala inhibits both in vitro and in vivo xenograft growth of pancreatic tumor cells by inducing apoptosis. BMC Cancer 2008;8:294.

64. Vadde R, Radhakrishnan S, Reddivari L, Vanamala JK. Triphala extract suppresses proliferation and induces apoptosis in human colon cancer stem cells via suppressing cMyc/cyclin D1 and elevation of Bax/Bcl-2 ratio. BioMed Res Int 2015;2015:649263.

65. Sandhya T, Lathika KM, Pandey BN, Mishra KP. Potential of traditional ayurvedic formulation, Triphala, as a novel anticancer drug. Cancer Lett 2006;231:206-214.

66. Phetkate P, Kummalue TYUP, Kietinun S. Significant increase in cytotoxic $\mathrm{T}$ lymphocytes and natural killer cells by triphala: A clinical phase I study. Evid Based Complement Altern Med 2012;2012:239856.

67. Gupta SK, Kalaiselvan V, Srivastava S, et al. Evaluation of anticataract potential of Triphala in selenite-induced cataract: In vitro and in vivo studies. J Ayurveda Integr Med 2010;1:280-286.

68. Kalaiselvan S, Rasool M. Triphala exhibits anti-arthritic effect by ameliorating bone and cartilage degradation in adjuvant-induced arthritic rats. Immunol Invest 2015;44: 411-426.

69. Kalaiselvan S, Rasool MK. The anti-inflammatory effect of triphala in arthritic-induced rats. Pharm Biol 2015;53:51-60.
70. Kalaiselvan S, Rasool MK. Triphala herbal extract suppresses inflammatory responses in LPS-stimulated RAW 264.7 macrophages and adjuvant-induced arthritic rats via inhibition of NF-kappaB pathway. J Immunotoxicol 2016; 13:509-525.

71. Reddy DB, et al. Chebulagic acid, a COX-LOX dual inhibitor isolated from the fruits of Terminalia chebula Retz., induces apoptosis in COLO-205 cell line. J Ethnopharmacol 2009; 124:506-512.

72. Baskaran UL, Martin SJ, Mahaboobkhan R, Prince SE. Protective role of Triphala, an Indian traditional herbal formulation, against the nephrotoxic effects of bromobenzene in Wistar albino rats. J Integr Med 2015;13:115121.

73. Varma SR, et al. Protective effects of triphala on dermal fibroblasts and human keratinocytes. PLoS One 2016;11: $\mathrm{e} 0145921$.

74. Yadav S, Gite S, Nilegaonkar S, Agte V. Effect of supplementation of micronutrients and phytochemicals to fructooligosaccharides on growth response of probiotics and E. coli. Biofactors 2011;37:58-64.

75. Tabasco R, et al. Effect of grape polyphenols on lactic acid bacteria and Bifidobacteria growth: Resistance and metabolism. Food Microbiol 2011;28:1345-1352.

76. Boto-Ordonez M, et al. High levels of Bifidobacteria are associated with increased levels of anthocyanin microbial metabolites: A randomized clinical trial. Food Funct 2014; 5:1932-1938

77. Jimenez N, Esteban-Torres M, Mancheno JM, et al. Tannin degradation by a novel tannase enzyme present in some Lactobacillus plantarum strains. Appl Environ Microbiol 2014;80:2991-2997.

78. Matoba Y, et al. Crystallographic and mutational analyses of tannase from Lactobacillus plantarum. Proteins 2013;81: 2052-2058.

79. Jimenez N, Curiel JA, Reveron I, et al. Uncovering the Lactobacillus plantarum WCFS1 gallate decarboxylase involved in tannin degradation. Appl Environ Microbiol 2013;79: 4253-4263.

Address correspondence to: Christine Tara Peterson, PhD Department of Family Medicine and Public Health UC San Diego School of Medicine Center of Excellence for Research and Training in Integrative Health 9500 Gilman Drive \#0725 La Jolla, CA 9093

E-mail: chpeterson@ucsd.edu 